\title{
Operator Method for Nonstationary Temperature Field Calculation in Thick-walled Cylindrical Heat Pipe Environment
}

\author{
Voldemārs Barkāns* \\ Latvian Maritime Academy
}

\begin{abstract}
The paper is dedicated to the exploration of one of the methods of renewable energy research. A significance lies in the economically efficient facilities; therefore, it is important to establish distribution of temperatures in the parts of facilities.

Considering a nonstationary process, the temperature field is described in the polar coordinate system by using Laplace's equation and corresponding mixed-type boundary data. The solution was obtained by the Laplace Transform Method, applying an integral function of complex variables. The inverse Laplace Transform and the original temperature are expressed as an integral. For the integration, a closed contour, which excludes branching and provides the integral of a function that is analytic, was employed. The Cauchy theorem was applied to the calculations. As a result, indefinite integrals were derived for the temperature estimate in the heat pipe cover and the surrounding environment, depending on the temperature of a fluid within the heat pipe.
\end{abstract}

Keywords - Laplace Transform, solar thermal collectors, thermal conductivity.

\section{INTRODUCTION}

Considering renewable energy generation methods [1], [2], the main problems encountered concern heat pipes. Solar thermal collectors and geothermal heat appliances widely employ heat pipes, situated indoors or outdoors, into the soil. It is essential to consider the effect of the heat pipe on the surrounding environment, as well as the effect of the surrounding environment on the heat pipe. Thus, the temperature field is calculated, depending on the fluid temperature within the heat pipe. One of the calculation methods is the Laplace Transform Method, which is widely employed by Carslaw H. S. and Jaeger J. C. [6], [7], and many other researchers.

\section{TEMPERATURE FIELD DESCRIPTION}

Nonstationary temperature field $T(r, \tau)$ in cylindrical heat pipe environment (Fig. 1), filled with a special fluid with constant temperature $T_{0}$, is described in a thermal conductivity equation [3]-[7] by using polar coordinates:

$$
\frac{\partial T(r, \tau)}{\partial \tau}=a\left(\frac{\partial^{2} T}{\partial r^{2}}+\frac{1}{r} \frac{\partial T}{\partial r}\right), \quad r \geq r_{0} ; \tau \geq 0,
$$

where $T(r, \tau)=\left\{\begin{array}{llr}T_{1}(r, \tau), & \text { if } & r_{0} \leq r \leq r_{1} \\ T_{2}(r, \tau), & \text { if } & r \geq r_{1}\end{array}\right.$

$a$ - coefficient of thermal conductivity, $\frac{\mathrm{m}^{2}}{\mathrm{~s}}$;

$$
a=\left\{\begin{array}{llr}
a_{1}, & \text { if } & r_{0} \leq r \leq r_{1} \\
a_{2}, & \text { if } & r \geq r_{1} .
\end{array}\right.
$$

At the boundary $r=r_{0}$, heat exchange between the fluid and the pipe occurs according to Newton's law:

$$
\lambda_{1} \frac{\partial T_{1}\left(r_{0}, \tau\right)}{\partial r}=-\alpha\left[T_{0}-T_{1}\left(r_{0}, \tau\right)\right]
$$

where $\alpha$-heat exchange coefficient, $\frac{\mathrm{W}}{\mathrm{m}^{2} \cdot \mathrm{K}}$;

$T_{0}$ - fluid temperature within the heat pipe, $\mathrm{K}$.

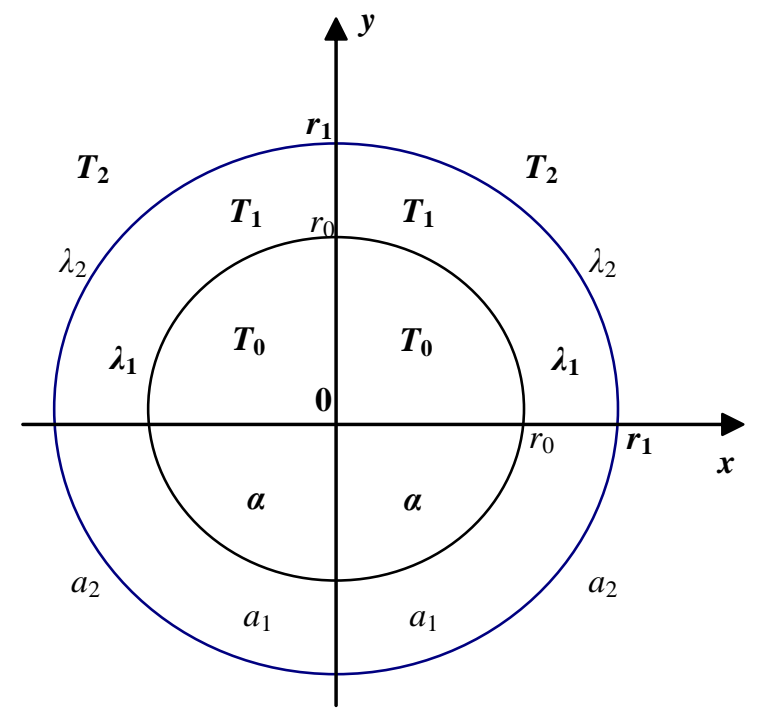

Fig. 1. Cross-section of a pipe.

At the boundary $r=r_{1}$, temperatures and heat flows have to be equal:

$$
T_{2}\left(r_{1}, \tau\right)=T_{1}\left(r_{1}, \tau\right)
$$

\footnotetext{
* Corresponding author.

E-mail address: voldemars.barkans@inbox.lv
} 


$$
\lambda_{2} \frac{\partial T_{2}\left(r_{1}, \tau\right)}{\partial r}=\lambda_{1} \frac{\partial T\left(r_{1}, \tau\right)}{\partial r},
$$

where $\lambda_{1}-$ thermal conductivity coefficient of a pipe cover, $\frac{\mathrm{W}}{\mathrm{m} \cdot \mathrm{K}}$;

$\lambda_{2}$ - thermal conductivity coefficient of soil, $\frac{\mathrm{W}}{\mathrm{m} \cdot \mathrm{K}} ;$

Initial temperature $\tau=0$ :

$$
T_{i}(r, 0)=T_{0 i}, \quad i=1,2
$$

At a large distance from the cylinder axis, the temperature is constant:

$$
T_{2}(\infty, \tau)=T_{\infty}
$$

and

$$
\frac{\partial T_{2}(\infty, \tau)}{\partial r}=0
$$

Dimensionless variables and parameters are introduced [3]:

$$
\begin{aligned}
& \frac{r}{r_{0}}=\rho ; \frac{r_{1}}{r_{0}}=\rho_{1} ; \frac{T_{i}}{T_{0}}=\Theta_{i} ; \frac{T_{0 i}}{T_{0}}=\Theta_{0 i} ; \frac{T_{\infty}}{T_{0}}=\Theta_{\infty} k^{2}=\frac{a_{1}}{a_{2}} ; \\
& \Lambda=\frac{\lambda_{1}}{\lambda_{2}} ; F=\frac{a_{1} \tau}{r_{0}^{2}} ; B i=\frac{\alpha r_{0}}{\lambda} ;
\end{aligned}
$$

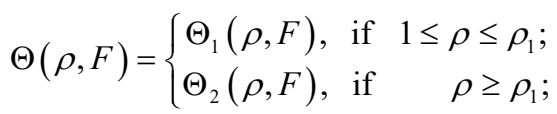

Thus, from (1)-(11), we obtain the following:

$$
\begin{gathered}
\frac{\partial \Theta_{1}(\rho, F)}{\partial F}=\frac{\partial^{2} \Theta_{1}(\rho, F)}{\partial \rho^{2}}+\frac{1}{\rho} \frac{\partial \Theta_{1}(\rho, F)}{\partial \rho} ; \\
k^{2} \frac{\partial \Theta_{2}(\rho, F)}{\partial F}=\frac{\partial^{2} \Theta_{2}(\rho, F)}{\partial \rho^{2}}+\frac{1}{\rho} \frac{\partial \Theta_{2}(\rho, F)}{\partial \rho} ; \\
\frac{\partial \Theta_{1}(1, F)}{\partial \rho}=-B i\left[1-\Theta_{1}(1, F)\right] ; \\
\Theta_{2}\left(\rho_{1}, F\right)=\Theta_{1}\left(\rho_{1}, F\right) ; \\
\frac{\partial \Theta_{2}\left(\rho_{1}, F\right)}{\partial \rho}=\Lambda_{1} \frac{\partial \Theta_{1}\left(\rho_{1}, F\right)}{\partial \rho} ; \\
\Theta_{i}(\rho, 0)=\Theta_{0 i} ; \quad i=1,2 ; \\
\Theta_{2}(\infty, F)=\Theta_{\infty} ; \\
\frac{\partial \Theta_{2}(\infty, F)}{\partial \rho}=0 .
\end{gathered}
$$

The equation is solved by using the Laplace Transform [6][12]:

$$
\bar{\Theta}_{i}(\rho, p)=\int_{0}^{+\infty} e^{p F} \Theta_{i}(\rho, F) d F
$$

By using the Laplace Transform, we obtain:

$$
\begin{gathered}
\frac{d^{2} \bar{\Theta}_{i}}{d \rho^{2}}+\frac{1}{\rho} \frac{d \bar{\Theta}_{i}}{d \rho}-p \bar{\Theta}_{i}=-\Theta_{01} \\
\frac{d^{2} \bar{\Theta}_{i}}{d \rho^{2}}+\frac{1}{\rho} \frac{d \bar{\Theta}_{i}}{d \rho}-p k^{2} \bar{\Theta}_{i}=-k^{2} \Theta_{02} \\
\frac{d \bar{\Theta}_{1}(1, p)}{d \rho}=-B i\left[\frac{1}{p}-\bar{\Theta}_{1}(1, p)\right]
\end{gathered}
$$

$$
\bar{\Theta}_{2}\left(\rho_{1}, p\right)=\bar{\Theta}_{1}\left(\rho_{1}, p\right)
$$

$$
\begin{gathered}
\frac{\partial \bar{\Theta}_{2}\left(\rho_{1}, p\right)}{\partial \rho}=\Lambda \frac{\partial \bar{\Theta}_{1}\left(\rho_{1}, p\right)}{\partial \rho} ; \\
\bar{\Theta}_{2}(\infty, p)=\frac{\Theta}{p} \\
\frac{\partial \bar{\Theta}_{2}(\infty, p)}{\partial \rho}=0
\end{gathered}
$$

The equations (21) and (22) are inhomogeneous Bessel differential equations [11]-[15]. The generic solution is obtained by the zeroth-order modified Bessel and Macdonald functions of the first and second kind $I_{0}(z)$ and $K_{0}(z)$ :

$$
\begin{gathered}
\bar{\Theta}_{1}(\rho, p)=C_{1} I_{0}(\rho \sqrt{p})+C_{2} K_{0}(\rho \sqrt{p})+\frac{\Theta_{01}}{p} \\
1 \leq \rho \leq \rho_{1} \\
\bar{\Theta}_{2}(\rho, p)=C_{3} I_{0}(k \rho \sqrt{p})+C_{4} K_{0}(k \rho \sqrt{p})+\frac{\Theta_{02}}{p} \\
\rho_{1} \leq \rho \leq+\infty
\end{gathered}
$$

The solution has to be exhaustive, if $\rho \rightarrow \infty$. Thus, from (29) we obtain the following:

$$
\bar{\Theta}_{2}(\rho, p)=C_{4} K_{0}(k \rho \sqrt{p})+\frac{\Theta_{02}}{p}
$$

Entering the boundary data (25)-(27) and equations [14] $I_{0}^{\prime}(z)=I_{1}(z), K_{0}^{\prime}(z)=-K_{1}(z)$, we obtain the equation for the calculation of the system coefficients $C_{1}, C_{2}, C_{4}$ : 


$$
\left\{\begin{array}{l}
C_{1}\left(\sqrt{p} I_{1}(\sqrt{p})-B i I_{0}(\sqrt{p})\right)- \\
-C_{2}\left(\sqrt{p} K_{1}(\sqrt{p})+B i K_{0}(\sqrt{p})\right)=\frac{B i}{p}\left(\Theta_{01}-1\right) ; \\
C_{1} \Lambda I_{1}\left(\rho_{1} \sqrt{p}\right)- \\
-C_{2} \Lambda K_{1}\left(\rho_{1} \sqrt{p_{2}}\right)+C_{4} k K_{1}\left(k \rho_{1} \sqrt{p}\right)=0 ; \\
C_{1} I_{0}\left(\rho_{1} \sqrt{p}\right)+ \\
+C_{2} K_{0}\left(\rho_{1} \sqrt{p}\right)-C_{4} K_{0}\left(k \rho_{1} \sqrt{p}\right)=\frac{\Theta_{02}-\Theta_{01}}{p} .
\end{array}\right.
$$

The determinant of the system (31) is as follows:

$$
\begin{aligned}
& \Delta\left(\rho_{1}, p\right)=\left[\sqrt{p} I_{1}(\sqrt{p})-B i I_{0}(\sqrt{p})\right] . \\
& \cdot\left[\Lambda K_{1}\left(\rho_{1} \sqrt{p}\right) K_{0}\left(k \rho_{1} \sqrt{p}\right)-k K_{1}\left(k \rho_{1} \sqrt{p}\right) K_{0}\left(\rho_{1} \sqrt{p}\right)\right]- \\
& -\left[\sqrt{p} K_{1}(\sqrt{p})+B i K_{0}(\sqrt{p})\right] . \\
& \cdot\left[\Lambda I_{1}\left(\rho_{1} \sqrt{p}\right) K_{0}\left(k \rho_{1} \sqrt{p}\right)+k K_{1}\left(k \rho_{1} \sqrt{p}\right) I_{0}\left(\rho_{1} \sqrt{p}\right)\right] .
\end{aligned}
$$

By solving a system of equations (31), the coefficients are calculated and the expression temperatures for the figures (28) and (29) are determined:

$$
\bar{\Theta}_{1}(\rho, p)=\frac{\Theta_{01}}{p}+\frac{\bar{\Theta}_{1}^{*}(\rho, p)}{p \Delta\left(\rho_{1}, p\right)},
$$

where

$$
\begin{aligned}
& \bar{\Theta}_{1}^{*}(\rho, p)=I_{0}(\rho \sqrt{p}) \cdot\left[B i\left(\Theta_{01}-1\right) \cdot\right. \\
& {\left[\Lambda K_{1}\left(\rho_{1} \sqrt{p}\right) K_{0}\left(k \rho_{1} \sqrt{p}\right)-k K_{1}\left(k \rho_{1} \sqrt{p}\right) K_{0}\left(\rho_{1} \sqrt{p}\right)\right]-} \\
& -k\left(\Theta_{02}-\Theta_{01}\right) K_{1}\left(k \rho_{1} \sqrt{p}\right) \cdot\left[\sqrt{p} K_{1}(\sqrt{p})+\right. \\
& \left.\left.+B I K_{0}(\sqrt{p})\right]\right]++K_{0}(\rho \sqrt{p}) \cdot\left[B i\left(\Theta_{01}-1\right) \cdot\right. \\
& \cdot\left[\Lambda I_{1}\left(\rho_{1} \sqrt{p}\right) K_{0}\left(\rho_{1} \sqrt{p}\right)+k K_{1}\left(k \rho_{1} \sqrt{p}\right) I_{0}\left(\rho_{1} \sqrt{p}\right)\right]- \\
& -k\left(\Theta_{02}-\Theta_{01}\right) K_{1}\left(k \rho_{1} \sqrt{p}\right) \cdot \\
& \left.\left.\cdot\left[\sqrt{p} I_{1}(\sqrt{p})-B i I_{0}(\sqrt{p})\right]\right]\right\} \\
& \bar{\Theta}_{2}(\rho, p)=\frac{\Theta_{02}}{p}+\Lambda B i \frac{\Theta_{01}-1}{\rho_{1}} \cdot \frac{K_{0}(k \rho \sqrt{p})}{p \sqrt{p} \Delta\left(\rho_{1}, p\right)}+ \\
& +\Lambda\left(\Theta_{02}-\Theta_{01}\right) \frac{\bar{\Theta}_{2}^{*}(\rho, p)}{p \Delta\left(\rho_{1}, p\right)},
\end{aligned}
$$

where

$$
\begin{aligned}
& \bar{\Theta}_{2}(\rho, p)=K_{0}(k \rho \sqrt{p}) \cdot \\
& \cdot\left\{I_{1}\left(\rho_{1} \sqrt{p}\right) \cdot\left[\sqrt{p} K_{1}(\sqrt{p})+B i K_{0}(\sqrt{p})\right]-\right. \\
& \left.-K_{1}\left(\rho_{1} \sqrt{p}\right) \cdot\left[\sqrt{p} I_{1}(\sqrt{p})-B i I_{0}(\sqrt{p})\right]\right\},
\end{aligned}
$$

or

$$
\bar{\Theta}_{i}(\rho, p)=\frac{\Theta_{0 i}}{p}+\overline{\bar{\Theta}}_{i}^{*}(\rho, p), \quad i=1,2 .
$$

The original temperature [6]-[8] is expressed as an integral:

$$
\Theta_{i}(\rho, F)=\Theta_{0 i}+\frac{1}{2 \pi j} \int_{\gamma-j \omega}^{\gamma+j \omega} e^{p F} \overline{\bar{\Theta}}_{i}^{*}(\rho, p) d p, j=\sqrt{-1} .
$$

The integral on the straight line $\operatorname{Re} p=\gamma$, where $p$ is a complex number $p=\gamma+j \omega$, can be determined [2], [3], [4] by the integrals on the line segment $\mathrm{AB}$ and the circle $p=\mathrm{Re}^{j \phi}$ arcs BB' $F$ and CA'A, along the upper edge EF and the lower edge $\mathrm{CD}$ of the section (Fig. 2), as well as along the inner circle $p=r e^{j \phi}$, which altogether form a closed contour. The integral on the line segment $\mathrm{AB}$ equals the sum of integrals on all the other parts of the contour, as the integral function is analytic within the contour. By calculating these integrals, if $R \rightarrow \infty$ and $r \rightarrow 0$, we obtain an integral that is necessary in the formula (36).

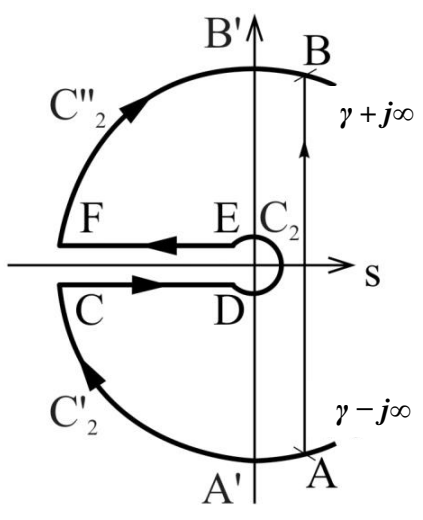

Fig. 2. Integration contour [6].

\section{Calculation of the Temperature of A PiPe Cover}

The following integral has to be calculated:

$$
\begin{aligned}
& \int_{\gamma-j \omega}^{\gamma+j \omega} e^{p F} \frac{\bar{\Theta}_{1}^{*}(\rho, p)}{p \Delta\left(\rho_{1}, p\right)} d p=\lim _{R \rightarrow \infty}\left(\int_{\mathrm{BB} F}+\int_{\mathrm{FE}}+\int_{\mathrm{DC}}+\right. \\
& \left.+\int_{\mathrm{DC}}+\int_{\mathrm{CA}^{\prime} \mathrm{A}}\right)+\lim _{r \rightarrow 0} \oint_{\mathrm{C}_{r}} .
\end{aligned}
$$

In the brackets of the equation (38), the boundary of both side integrals, if $R \rightarrow+\infty$, according to Jordan's lemma [8], equals zero: 


$$
\lim _{R \rightarrow \infty} \int_{\mathrm{BB} F}=0 ; \quad \lim _{R \rightarrow \infty} \int_{\mathrm{CA}^{\prime} \mathrm{A}}=0 .
$$

Thus, we calculate the value of the system (31) determinant (32) on the line segment EF:

$$
p=u^{2} e^{j \pi}=-u^{2} ; \sqrt{p}=u e^{j \frac{\pi}{2}}=j u ; d p=2 u e^{j \pi} d u .
$$

By adding (41) to the formula (32), we obtain the following:

$$
\begin{gathered}
\Delta_{\mathrm{EF}}\left(\rho_{1},-u^{2}\right)=\left[j u I_{1}(j u)-B i I_{0}(j u)\right] \cdot \\
\cdot\left[\Lambda K_{1}\left(\rho_{1} j u\right) K_{0}\left(k \rho_{1} j u\right)-\right. \\
\left.-k K_{1}\left(k \rho_{1} j u\right) K_{0}\left(\rho_{1} j u\right)\right]-\left[j u K_{1}(j u)+B i K_{0}(j u)\right] . \\
\cdot\left[\Lambda I_{1}\left(\rho_{1} j u\right) K_{0}\left(k \rho_{1} j u\right)+k K_{1}\left(k \rho_{1} j u\right) I_{0}\left(\rho_{1} j u\right)\right] .
\end{gathered}
$$

The formulae [6], [7], [11] are applied to the Bessel functions with a purely imaginary argument:

$$
\begin{aligned}
& I_{v}( \pm z)=e^{ \pm j v \frac{\pi}{2}} J_{v}(z) \\
& K_{v}( \pm z)= \pm j \frac{\pi}{2} e^{\mp j v \frac{\pi}{2}}\left[-J_{v}(z) \pm j Y_{v}(z)\right]
\end{aligned}
$$

Here, $J_{0}(z), Y_{0}(z), J_{1}(z), Y_{1}(z)$ correspond to the zeroth order functions of the first and second kind and to the Bessel functions of the first kind. Bessel functions are then added to the system determinant equation (32), and after the modifications we obtain the following equation:

$$
\Delta_{\mathrm{EF}}\left(\rho_{1},-u^{2}\right)=\frac{\pi^{2}}{4}[\phi(u)+j \psi(u)],
$$

where

$$
\begin{gathered}
\phi(u)=s_{1}(u) \cdot\left[k J_{1}\left(k \rho_{1} u\right) Y_{0}\left(\rho_{1} u\right)-\Lambda J_{0}\left(k \rho_{1} u\right) Y_{1}\left(\rho_{1} u\right)\right]+ \\
+s_{2}(u) \cdot\left[\Lambda J_{0}\left(k \rho_{1} u\right) J_{1}\left(\rho_{1} u\right)-k J_{1}\left(k \rho_{1} u\right) J_{0}\left(\rho_{1} u\right)\right] ; \\
\psi(u)=s_{2}(u) \cdot\left[k Y_{1}\left(k \rho_{1} u\right) J_{0}\left(\rho_{1} u\right)-\Lambda Y_{0}\left(k \rho_{1} u\right) J_{1}\left(\rho_{1} u\right)\right]+ \\
+s_{1}(u) \cdot\left[\Lambda Y_{0}\left(k \rho_{1} u\right) Y_{1}\left(\rho_{1} u\right)-k Y_{1}\left(k \rho_{1} u\right) Y_{0}\left(\rho_{1} u\right)\right] \\
s_{1}(u)=u J_{1}(u)+B i J_{0}(u) \\
s_{2}(u)=u Y_{1}(u)+B i Y_{0}(u)
\end{gathered}
$$

The value of the function $\bar{\Theta}_{1}^{*}(\rho, p)$ is then calculated on the line segment EF:

$$
\begin{gathered}
\bar{\Theta}_{1 \mathrm{EF}}^{*}\left(\rho,-u^{2}\right)=\left\{I _ { 0 } ( \rho j u ) \cdot \left[B i\left(\Theta_{01}-1\right) \cdot\right.\right. \\
\cdot\left[\Lambda K_{1}\left(\rho_{1} j u\right) K_{0}\left(k \rho_{1} j u\right)-k K_{1}\left(k \rho_{1} j u\right) .\right. \\
\left.\cdot K_{0}\left(\rho_{1} j u\right)\right]-k\left(\Theta_{02}-\Theta_{01}\right) K_{1}\left(k \rho_{1} j u\right) .
\end{gathered}
$$

$$
\begin{gathered}
\left.\cdot\left[j u K_{1}(j u)+B i K_{0}(j u)\right]\right]+K_{0}(\rho j u) \cdot \\
\cdot\left[\operatorname { B i } ( \Theta _ { 0 1 } - 1 ) \cdot \left[\Lambda I_{1}\left(\rho_{1} j u\right) K_{0}\left(\rho_{1} j u\right)+k \cdot\right.\right. \\
\left.\cdot K_{1}\left(k \rho_{1} j u\right) I_{0}\left(\rho_{1} j u\right)\right]-k\left(\Theta_{02}-\Theta_{01}\right) \cdot \\
\left.\left.\cdot K_{1}\left(k \rho_{1} j u\right) \cdot\left[\sqrt{p} I_{1}(j u)-B i I_{0}(j u)\right]\right]\right\} .
\end{gathered}
$$

By using the formulae for the Bessel functions with purely imaginary argument, we obtain the following:

$$
\bar{\Theta}_{1 \mathrm{EF}}^{*}\left(\rho,-u^{2}\right)=\frac{\pi^{2}}{4}\left[\phi_{1}(u)+j \psi_{1}(u)\right],
$$

where

$$
\begin{gathered}
\phi_{1}(\rho, u)=B i\left(\Theta_{01}-1\right) \cdot\left[\Lambda J_{0}\left(k \rho_{1} u\right) \cdot\right. \\
\cdot\left[J_{0}(\rho u) Y_{1}\left(\rho_{1} u\right)-Y_{0}(\rho u) J_{1}\left(\rho_{1} u\right)\right]- \\
\left.-k J_{1}\left(k \rho_{1} u\right)\left[J_{0}(\rho u) Y_{0}\left(\rho_{1} u\right)-Y_{0}(\rho u) J_{0}\left(\rho_{1} u\right)\right]\right]+ \\
+k\left(\Theta_{02}-\Theta_{01}\right) J_{1}\left(k \rho_{1} u\right) \cdot\left[Y_{0}(\rho u) s_{1}(u)-J_{0}(\rho u) s_{2}(u)\right] ; \\
\psi_{1}(\rho, u)=B i\left(\Theta_{01}-1\right) \cdot\left[\Lambda Y_{0}\left(k \rho_{1} u\right) \cdot\right. \\
\cdot\left[Y_{0}(\rho u) J_{1}\left(\rho_{1} u\right)-J_{0}(\rho u) Y_{1}\left(\rho_{1} u\right)\right]- \\
+k Y_{1}\left(k \rho_{1} u\right)\left[Y_{0}(\rho u) J_{0}\left(\rho_{1} u\right)-J_{0}(\rho u) Y_{0}\left(\rho_{1} u\right)\right]+ \\
+k\left(\Theta_{02}-\Theta_{01}\right) Y_{1}\left(k \rho_{1} u\right)\left[J_{0}(\rho u) s_{2}(u)-Y_{0}(\rho u) s_{1}(u)\right] .
\end{gathered}
$$

By using (44) and (50), we obtain an integral on the line segment EF, if $R \rightarrow+\infty$ :

$$
\begin{gathered}
I_{\mathrm{EF}}=2 \int_{0}^{+\infty} e^{-u^{2} F} \frac{\phi(u) \phi_{1}(u)+\psi(u) \psi_{1}(u)}{\phi^{2}(u)+\psi^{2}(u)} \frac{d u}{u}+ \\
+2 j \int_{0}^{+\infty} e^{-u^{2} F} \frac{\left[\phi(u) \psi_{1}(u)-\psi(u) \phi_{1}(u)\right]}{\phi^{2}(u)+\psi^{2}(u)} \frac{d u}{u}
\end{gathered}
$$

On the line segment CD:

$$
p=u^{2} e^{-j \pi}=-u^{2} ; \quad \sqrt{p}=-j u ; d p=2 u e^{-j \pi} d u
$$

Analogous to the line segment EF, by using formulae [6] for Bessel functions with purely imaginary argument, we obtain the system (31) determinant (32) on the line segment CD:

$$
\Delta_{\mathrm{CD}}\left(\rho_{1},-u^{2}\right)=\frac{\pi^{2}}{4}[\phi(u)-j \psi(u)],
$$


and the function $\bar{\Theta}_{1}^{*}(\rho, p)$ :

$$
\bar{\Theta}_{1 \mathrm{CD}}^{*}\left(\rho,-u^{2}\right)=\frac{\pi^{2}}{4}\left[\phi_{1}(u)-j \psi_{1}(u)\right] .
$$

By using the equations (44) and (57), we obtain an integral on the line segment $\mathrm{CD}$, if $R \rightarrow+\infty$ :

$$
\begin{aligned}
I_{\mathrm{CD}}=2 \int_{0}^{+\infty} e^{-u^{2} F} \frac{\phi(u) \phi_{1}(u)+\psi(u) \psi_{1}(u)}{\phi^{2}(u)+\psi^{2}(u)} \frac{d u}{u}- \\
\quad-2 j \int_{0}^{+\infty} e^{-u^{2} F} \frac{\left[\phi(u) \psi_{1}(u)-\psi(u) \phi_{1}(u)\right]}{\phi^{2}(u)+\psi^{2}(u)} \frac{d u}{u} .
\end{aligned}
$$

In order to calculate the boundary $\lim _{r \rightarrow 0} \oint_{\mathrm{C}_{r}} e^{p F} \frac{\bar{\Theta}_{1}^{*}(\rho, p)}{p \Delta\left(\rho_{1}, p\right)} d p$ of an integral on the inner circle, where

$$
p=r e^{j \phi} ; d p=r e^{j \phi} j d \phi,
$$

first, the boundary has to be determined:

$$
\lim _{r \rightarrow 0} \frac{\bar{\Theta}_{1}^{*}(\rho, p)}{\Delta\left(\rho_{1}, p\right)}=1-\Theta_{01} .
$$

In the boundary data calculation, the following patterns are to be considered [15]:

If $0<x<<1$, then $I_{0}(x) \cong 1 ; I_{1}(x) \cong \frac{x}{2}$;

$$
K_{0}(x) \cong \ln \frac{2}{\gamma x} ; K_{1}(x)=\frac{1}{x} ;
$$

if $x>>1$, then $I_{v}(x) \cong \frac{e^{x}}{\sqrt{2 \pi x}}$;

$$
K_{v}(x) \cong e^{-x} \sqrt{\frac{\pi}{2 x}} .
$$

We obtain the following:

$$
\lim _{r \rightarrow 0} \oint_{\mathrm{C}_{r}} e^{p F} \frac{\bar{\Theta}_{1}^{*}(\rho, p)}{p \Delta\left(\rho_{1}, p\right)} d p=2 \pi j\left(1-\Theta_{01}\right) .
$$

By applying the estimated integrals (40), (54), (57), and (63) to the formula (39), we obtain a dimensionless temperature expression in the pipe cover $\left(1 \leq \rho \geq \rho_{1}\right)$ :

$$
\begin{aligned}
\Theta_{1}(\rho, F) & =1+ \\
& +\frac{2}{\pi} \int_{0}^{+\infty} e^{-u^{2} F} \frac{\phi(u) \psi_{1}(\rho, u)-\psi(u) \phi_{1}(\rho, u)}{\phi^{2}(u)+\psi^{2}(u)} \frac{d u}{u} .
\end{aligned}
$$

By determining the indefinite integral in the formula (64), we obtain the real pipe cover temperature.

\section{SOIL TEMPERATURE CALCULATION}

In the integral (39), if $i=2$, first we have to calculate the following:

$$
\begin{gathered}
\int_{\gamma-j \omega}^{\gamma+j \omega} e^{p F} \frac{K_{0}(k \rho \sqrt{p})}{p \sqrt{p} \Delta\left(\rho_{1}, p\right)} d p=\lim _{R \rightarrow \infty}\left(\int_{\mathrm{BBF}}+\int_{\mathrm{FE}} e^{p F}+\int_{\mathrm{DC}}+\right. \\
\left.+\int_{\mathrm{CA} A}\right)+\lim _{r \rightarrow 0} \oint_{\mathrm{C}_{r}} .
\end{gathered}
$$

In the equation (65), the boundary of both side integrals, if $R \rightarrow+\infty$, according to Jordan's lemma [8], equals zero:

$$
\lim _{R \rightarrow \infty} \int_{\text {BBF }}=0 ; \lim _{R \rightarrow \infty} \int_{\text {CA'A }}=0 .
$$

The value of the system (31) determinant (32) on the line segment EF is calculated with the formula (41). The function $K_{0}(k \rho \sqrt{p})$, if $\sqrt{p}=j u$, is calculated by employing the formula (44); thus we obtain the following:

$$
K_{0}(k \rho j u)=\frac{\pi}{2}\left[-Y_{0}(k \rho u)-j J_{0}(k \rho u)\right]
$$

By combining (45) and (67), we obtain the integral:

$$
\begin{gathered}
\int_{\mathrm{FE}} e^{p F} \frac{K_{0}(k \rho \sqrt{p})}{p \sqrt{p} \Delta\left(\rho_{1}, p\right)} d p= \\
=\frac{4}{\pi} \int_{0}^{+\infty} e^{-u^{2} F} \frac{Y_{0}(k \rho u) \psi(u)-J_{0}(k \rho u) \phi(u)}{\phi^{2}(u)+\psi^{2}(u)} \frac{d u}{u^{2}}+ \\
+\frac{4}{\pi} j \int_{0}^{+\infty} e^{-u^{2} F} \frac{\left[Y_{0}(k \rho u) \phi(u)+J_{0}(k \rho u) \psi(u)\right]}{\phi^{2}(u)+\psi^{2}(u)} \frac{d u}{u^{2}} .
\end{gathered}
$$

Similarly, we obtain an integral on the line segment CD:

$$
\begin{gathered}
\int_{\mathrm{DC}} e^{p F} \frac{K_{0}(k \rho \sqrt{p})}{p \sqrt{p} \Delta\left(\rho_{1}, p\right)} d p= \\
=\frac{4}{\pi} \int_{0}^{+\infty} e^{-u^{2} F} \frac{Y_{0}(k \rho u) \psi(u)-J_{0}(k \rho u) \phi(u)}{\phi^{2}(u)+\psi^{2}(u)} \frac{d u}{u^{2}}- \\
-\frac{4}{\pi} j \int_{0}^{+\infty} e^{-u^{2} F} \frac{\left[Y_{0}(k \rho u) \phi(u)+J_{0}(k \rho u) \psi(u)\right]}{\phi^{2}(u)+\psi^{2}(u)} \frac{d u}{u^{2}} .
\end{gathered}
$$

By using the equations (61) and (63), the boundary is estimated:

$$
\lim _{r \rightarrow 0} \frac{K_{0}(k \rho \sqrt{p})}{\sqrt{p} \Delta\left(\rho_{1}, p\right)}=-\frac{\rho_{1}}{\Lambda B i} .
$$


And the integral on the inner circle is determined, where

$$
\begin{gathered}
p=r e^{j \phi} ; \quad d p=r e^{j \phi} j d \phi ; \\
\lim _{r \rightarrow 0} \oint_{\mathrm{C}_{r}} e^{p F} \frac{K_{0}(k \rho \sqrt{p})}{p \sqrt{p} \Delta\left(\rho_{1}, p\right)} d p=-2 \pi j \frac{\rho_{1}}{\Lambda B i} .
\end{gathered}
$$

The interim values (66), (69)-(71) are included in the equation (65) to obtain:

$$
\int_{\gamma-j \omega}^{\gamma+j \omega} e^{p F} \frac{K_{0}(k \rho \sqrt{p})}{p \sqrt{p} \Delta\left(\rho_{1}, p\right)} d p=-\frac{\rho_{1}}{\Lambda B i} .
$$

Now we calculate the second integral (39):

$$
\begin{gathered}
\int_{\gamma-j \omega}^{\gamma+j \omega} e^{p F} \frac{\bar{\Theta}_{2}^{*}(\rho, p)}{p \Delta\left(\rho_{1}, p\right)} d p==\lim _{R \rightarrow \infty}\left(\int_{\mathrm{BB} F}+\int_{\mathrm{FE}}+\right. \\
\left.+\int_{\mathrm{DC}}+\int_{\mathrm{CA}^{\prime} \mathrm{A}}\right)+\lim _{r \rightarrow 0} \oint_{\mathrm{C}_{r}} .
\end{gathered}
$$

In the equation (74), the boundary of both side integrals, if $R \rightarrow+\infty$, according to Jordan's lemma [4], equals zero:

$$
\lim _{R \rightarrow \infty} \int_{\mathrm{BB}^{\prime} \mathrm{F}}=0 ; \quad \lim _{R \rightarrow \infty} \int_{\mathrm{CA}^{\prime} \mathrm{A}}=0 .
$$

On the line segment EF (41), the system (31) determinant (32) value is determined by the formula (45), whereas the function $K_{0}(k \rho \sqrt{p})$, if $\sqrt{p}=j u$, is calculated by the formula (44). We obtain an integral on the line segment EF, if $R \rightarrow+\infty$ :

$$
\begin{gathered}
I_{\mathrm{EF}}=\int_{\mathrm{FE}} e^{p F} \frac{\bar{\Theta}_{2}^{*}(\rho, p)}{p \Delta\left(\rho_{1}, p\right)} d p= \\
=2 \int_{0}^{+\infty} e^{-u^{2} F} \frac{\left[Y_{0}(k \rho u) \psi(u)-J_{0}(k \rho u) \phi(u)\right]}{\phi^{2}(u)+\psi^{2}(u)} \phi^{*}(u) \frac{d u}{u}- \\
-2 j \int_{0}^{+\infty} e^{-u^{2} F} \frac{Y_{0}(k \rho u) \phi(u)+J_{0}(k \rho u) \psi(u)}{\phi^{2}(u)+\psi^{2}(u)} \phi^{*}(u) \frac{d u}{u}
\end{gathered}
$$

where

$$
\phi^{*}(u)=Y_{1}\left(\rho_{1} u\right) s_{1}(u)-J_{1}\left(\rho_{1} u\right) s_{2}(u)
$$

On the line segment CD (55), the system (31) determinant value (32) is determined by using the formula (56) and function $K_{0}(k \rho \sqrt{p})$, if $\sqrt{p}=-j u$, is calculated by the formula (45).
We obtain an integral on a line segment $\mathrm{CD}$, if $R \rightarrow+\infty$ :

$$
\begin{aligned}
& I_{\mathrm{DC}}==2 \int_{0}^{+\infty} e^{-u^{2} F} \frac{\left[J_{0}(k \rho u) \phi(u)-Y_{0}(k \rho u) \psi(u)\right]}{\phi^{2}(u)+\psi^{2}(u)} \phi^{*}(u) \frac{d u}{u}+ \\
& +2 j \int_{0}^{+\infty} e^{-u^{2} F} \frac{Y_{0}(k \rho u) \phi(u)+J_{0}(k \rho u) \psi(u)}{\phi^{2}(u)+\psi^{2}(u)} \phi^{*}(u) \frac{d u}{u}
\end{aligned}
$$

In order to calculate the boundary $\lim _{r \rightarrow 0} \oint_{\mathrm{C}_{r}} e^{p F} \frac{\bar{\Theta}_{2}^{*}(\rho, p)}{p \Delta\left(\rho_{1}, p\right)} d p$ of the integral on the inner circle (71), first, the boundary should be determined:

$$
\lim _{r \rightarrow 0} \frac{\bar{\Theta}_{2}^{*}(\rho, p)}{\Delta\left(\rho_{1}, p\right)}=-\frac{1}{\Lambda}
$$

We obtain the following:

$$
\lim _{r \rightarrow 0} \oint_{C_{r}} e^{p F} \frac{\bar{\Theta}_{2}^{*}(\rho, p)}{p \Delta\left(\rho_{1}, p\right)} d p=-\frac{2 \pi}{\Lambda} j .
$$

The obtained integrals (69), (70), (76)-(78), and (80) are then inserted in the equations (65) and (74). By using the equation (33), we can calculate the dimensionless temperature outside the heat $\operatorname{pipe}\left(\rho \geq \rho_{1}\right)$ :

$$
\begin{gathered}
\Theta_{2}(\rho, F)=1+\Lambda B i \frac{\Theta_{01}-1}{\rho_{1}} . \\
\cdot \frac{4}{\pi^{2}} \int_{0}^{+\infty} e^{-u^{2} F} \frac{\phi(u) Y_{0}(\rho u)+\psi(u) J_{0}(\rho u)}{\phi^{2}(u)+\psi^{2}(u)} \frac{d u}{u^{2}}+ \\
+\Lambda\left(\Theta_{02}-\Theta_{01}\right) \cdot \\
\cdot \frac{2}{\pi} \int_{0}^{+\infty} e^{-u^{2} F} \frac{\phi(u) Y_{0}(\rho u)+\psi(u) J_{0}(\rho u)}{\phi^{2}(u)+\psi^{2}(u)} \phi^{*}(u) \frac{d u}{u} .
\end{gathered}
$$

By determining the indefinite integrals in the formula (81), we obtain the real ambient temperature.

\section{CONCLUSION}

Thermal conductivity equation has been solved in polar coordinates, using the inner surface boundary conditions of the third kind and the contact conditions on the outer surface. The initial temperature is known. As a result, formulae for the temperature calculation in the heat pipe cover and surrounding environment have been devised provided that the fluid temperature within the heat pipe is known in advance. 


\section{REFERENCES}

[1] V. Barkāns, et al., "Nonstationary Heat conduction process on a Solar Collector," in 9th Int. Conf. Maritime transport and infrastructure, Riga: Latvian Maritime Academy, 2007, pp. 214-225.

[2] V. Barkāns, P. Šipkovs and M. Vanags, "Temperature Field in the Heatexchanger of a hot water Accumulator," in $10^{\text {th }}$ Int. Conf. Maritime transport and Infrastructure, Riga: Latvian Maritime Academy, 2008, pp. 221-225.

[3] V. P. Isachenko, V. A. Osipova and A. S. Sukomel, Teploperedacha, Moscow: Energija, 1969, p. 440. (in Russian)

[4] E. M. Kartashow, Analiticheskije metodi v teorii teploprovodnosti tverdix tel, Moscow: Vishaja shkola, 2001, p. 550. (in Russian)

[5] A. V. Likov, Teplomassoobmen, Moscow: Energija, 1972, p. 560. (in Russian)

[6] H. S. Carslaw and J. C. Jaeger, Teploprovodnostj tverdih tel, Moscow: Nauka, 1964, p. 487. (in Russian)

[7] H. S. Carslaw and J. C. Jaeger, Operacionnie metodi v prikladoj matematike, Moscow: IL, 1948, p. 293. (in Russian)

[8] M. A. Lavrentjev and B. V. Šabat, Metodi teorii funkcij kompleksnogo peremennogo, Moscow: Nauka, 1958, p. 678. (in Russian)

[9] N. S. Košljakov, E. B. Gliner and M. M. Smirnov, Osnovnije differenciljnije uravnenija matematitcheskoj fiziki, Moscow: Fizmat-giz, 1962, p. 768. (in Russian)

[10] A. N. Tihonov and A. A. Samarskij, Uuravnenija matematitcheskoj fiziki, Moscow: Nauka, 1956, p. 724. (in Russian)

[11] E. Riekstiņš, Matematiskās fizikas metodes, Riga: Zvaigzne, 1969, p. 620. (in Latvian)

[12] G. Doetsch, Rukovodstvo k praktitcheskomu primeneniju preobrazovanija Laplassa $i$ Z-preobrazovanija, Moscow: Nauka, 1971, p. 288. (in Russian)

[13] G. N. Watson, Teorija besselevih funkcij, Moscow: IL, 1949, p. 787. (in Russian)

[14] A. Gray and G. B. Mathews, Funkcii Besselja i ih priloženija $k$ fizike I mehanike, Moscow: IL, 1953, p. 372. (in Russian)

[15] E. Janke, F. Emde and F. Losch, Specialjnije funkcii, formuli, grafiki, tablici, Moscow: Fizmat-giz, 1968, p. 344. (in Russian)

[16] B. Key, "Heat Recovery from Chilled Water Systems," Carrier, USA, 2008 , p. 20

[17] K. D. Rafferty, "Absorbtion refrigeration. Geo-Heat Center Klamath Falls,” OR 97601, 2009, pp. 299-306.

[18] S. V. Patancar and D. B. Spalding, "A calculation procedure for heat, mass and momentum transfer in three-dimensional parabolic flows," Int. J. of Heat and Mass Transfer, vol. 15, issue 10, p. 1787, 1972. http://dx.doi.org/10.1016/0017-9310(72)90054-3

[19] K. Sopian, et al., "Performance of a non-metallic unglazed solar water heater with integrated storage system," Renewable energy, vol. 29, issue 9, pp. 1421-1430, 2004. http://dx.doi.org/10.1016/j.renene.2004.01.002

[20] R. S. Gorla, "Finite element analysis of a flat plate solar collector," Finite Elements in Analysis and Design, vol. 24, issue 4, pp. 283-290, 1997. http://dx.doi.org/10.1016/s0168-874x(96)00067-4

[21] O. Turgut and N. Onur, "Three-dimensional numerical and experimental study of forced convection heat transfer on solar collector surface," International Communication in Heat and Mass Transfer, vol. 36, pp. 274 279, 2009. http://dx.doi.org/10.1016/j.icheatmasstransfer.2008.10.017 